\title{
TTSH and NCID Radiology Services in COVID-19
}

\section{Dear Editor,}

The impact of COVID-19 pandemic on radiology is significant. It has resulted in alterations to layouts, workflow and protocols of a radiology department. Much of these have been thoroughly documented in recent articles. ${ }^{1,2}$

Singapore has more than 46,000 confirmed cases of COVID-19 infection with 27 deaths, while worldwide numbers are at more than 13.3 million cases with more than 580,000 deaths. Worldwide numbers continue unabated with a rising trend. ${ }^{2}$

The National Centre of Infectious Disease (NCID), where the majority of COVID-19 patients in Singapore are treated, is located within the Tan Tock Seng Hospital (TTSH) campus. NCID operates a satellite radiology service equipped with radiography units, ultrasound machines, a CT scanner and a fluoroscopy suite dedicated to management of COVID-19 patients. It is staffed by the main radiology department located in TTSH.

While most radiology departments in other hospitals have to manage non-COVID-19 and COVID-19 patients within the same location, having a satellite radiology suite in a dedicated infectious disease facility such as NCID confers several advantages in the COVID-19 pandemic: (1) COVID-19 patients and suspected cases who require radiological investigations or procedures need not be transported to the main radiology department in TTSH, reducing risks of transmission to other patients; (2) scan rooms placed in negative pressure aids in reducing transmission; (3) reduced exposure of COVID-19 patients to the radiology staff in the main radiology department; and (4) reduced disruption to 'business-as-usual'(BAU) workflow within the main radiology department.

Radiographers are rostered between the TTSH main radiology department for BAU operations, and the NCID satellite radiology service for pandemic operations. Before the pandemic, only a skeletal team of 2-3 radiographers were needed to operate in NCID. During the pandemic, a larger team of 6-8 radiographers are required to meet the increased patient load, particularly for $\mathrm{CT}$ and $\mathrm{X}$-rays studies, putting a strain on manpower. However, as non-essential radiological investigations are cancelled or postponed, the reduction in BAU workload allows for manpower diversions.
For radiographers as frontline staff in direct contact with patients, adherence to infectious disease protocols is paramount. Radiographers rostered to NCID are required to don a new set of full personal protective equipment (PPE), which includes an N95 mask, goggles, shower cap, gloves and gown when attending to each patient. Deep cleaning of the station is performed after each patient, which takes approximately 20-30 minutes. Deep cleaning entails thorough wipe down utilising Biospot, a chlorine disinfectant. For urgent cases requiring a fast turnaround, only cleaning of contact points is performed. This can be done without significant downtime and is possible only in a dedicated infectious disease facility, where the risk of cross-contamination between patient and staff is controlled. Further, to mitigate the potential risk of infection and cross-contamination between the NCID and main radiology department in TTSH, radiographers are assigned 8-hour shifts per day for 2 weeks in NCID.

For radiologists, there is similar need to cope with both BAU and pandemic operations. As screening for suspected cases and close contacts of COVID-19 patients are done in NCID, the number of chest X-rays performed between January and April 2020 has more than doubled compared with the same period in 2019. There is a need for rapid turnaround for reporting of these chest $X$-rays, to allow prompt discharge from the screening station.

To cope with surge in cases, 2-3 radiologists are assigned daily to report these screening chest X-rays from NCID, which can amount to several hundred a day. To boost turnaround, a team from the TTSH radiology department has worked to develop an artificial intelligence (AI) software capable of identifying lung changes suggestive of COVID-19 infection on screening chest $\mathrm{X}$-rays. These X-rays are to be flagged for more urgent reporting, which expedites management and patient isolation. Results for a recent AI study for COVID-19 identification using chest X-rays was found to have $78 \%$ sensitivity and $82 \%$ specificity. ${ }^{4}$

Meanwhile, essential imaging such as pre-operative imaging, cancer imaging for diagnosis and follow-up continues. The reported lack of imaging capacity due to deep cleaning/spacing of patients and increased workload during the COVID-19 pandemic has not affected our institution due to the abovementioned arrangements. 
Radiologists were also allocated into teams and location, limiting interaction for infection control. Furthermore, what was unique in the arrangement was the implementation of a small trial group of radiologists who were working from home/off-site from the hospital. The radiologists were to adhere to the stipulated IT policies and reporting standards, and were provided with the necessary equipment. The initiative in its trial state has shown promising results. While the teleradiology scene in Singapore has been established, ${ }^{5}$ emergence of COVID-19 has demonstrated the additional potential benefit of teleradiology in infection control.

Although workflow and protocols have been established to screen high-risk patients, undiagnosed COVID-19 patients may present at the TTSH main radiology department. This is not unexpected given the increasing use of imaging as part of patient management coupled with the fact that patients with COVID-19 may be asymptomatic in the first few days of infection. For example, a 68-year-old man was admitted to the general ward in TTSH for abdominal pain and underwent an abdominopelvic CT study as part of evaluation. Groundglass changes were seen in the lung bases. The patient had no respiratory symptoms or recent travel to high-risk countries, nor was he a close contact of a confirmed or suspect case. He was therefore deemed low-risk for COVID-19 at the time of admission. The total time he had spent in the department was approximately 30 minutes. This included 5 minutes in the CT scan room and 25 minutes in the waiting area. In view of incidental CT scan findings, he underwent COVID-19 testing, was confirmed positive two days later and immediately isolated.

In this case, the main risk of disease transmission was to the staff directly attending to the patient (nurses, radiographers and porters) and other patients within the department. It is recommended that a contingency protocol be prepared for such situations to determine if staff isolation or contact tracing is required. Given that the staff adhered to the necessary PPE, the already implemented safe distancing measures and the transient nature of contact (less than 30 minutes), the risk of infection was deemed low. Staff were able to continue working and monitor diligently for respiratory symptoms and fever. This prevented manpower disruption. Deep cleaning of used equipment and rooms is performed upon notification of patients' diagnosis. With physical distancing measures in place, and cancellation/postponement of non-essential imaging studies resulting in relatively small number of patients within the department, the risk of transmission to other patients within the department is deemed very low and contact tracing need not be performed.
Despite having to modify workflow and stretch manpower staffing for both BAU and pandemic operations between TTSH and NCID, protocols have also been established to reduce risk of cross-infections between staff and patients. It is reassuring that no radiology staff and BAU patients had required isolation or were diagnosed with COVID-19 during course of work or as a result of a department visit as of mid-July 2020.

Lessons learned from severe acute respiratory syndrome (SARS) epidemic in $2003^{6,7}$ and H1N1 pandemic in 2009 were incorporated into the physical design and construction of NCID as well as shaped infectious disease protocols and management. Ability to decant COVID-19 patients to the NCID satellite radiology service for imaging studies or procedures is advantageous in minimising downtime in BAU operations at the TTSH main radiology department. However, ultimately, compliance to infectious disease protocols is crucial, not only for personal and patient safety, but also to minimise disruption to manpower.

\section{REFERENCES}

1. Tan BP, Lim KC, Goh YG, et al. Radiology Preparedness in the Ongoing Battle against COVID-19: Experiences from Large to Small Public Hospitals in Singapore. Radiol Cardiothorac Imaging 2020;2:10.1148/ryct.2020200140.

2. World Health Organization. Coronavirus disease (COVID-19): situation report, 178, 2020. Available at: https://apps.who.int/iris/ handle/10665/333306. Accessed on 17 July 2020.

3. Cheng LT, Chan LP, Tan BH, et al. Déjà Vu or Jamais Vu? How the Severe Acute Respiratory Syndrome Experience Influenced a Singapore Radiology Department's Response to the Coronavirus Disease (COVID-19) Epidemic. AJR Am J Roentgenol 2020; 214:1206-10.

4. Castiglioni I, Ippolito D, Interlenghi M, et al. Artificial intelligence applied on chest X-ray can aid in the diagnosis of COVID-19 infection: a first experience from Lombardy, Italy. medRxiv 2020. DOI: https://doi.org/10.1101/2020.04.08.20040907. Accessed on 17 July 2020.

5. Cheng LT, Ng SE. Teleradiology in Singapore - Taking Stock and Looking Ahead. Ann Acad Med Sing 2006;35:552-6.

6. Tai DY. SARS: How to Manage Future Outbreaks? Ann Acad Med Singap 2006;35:368-73.

7. Goh KT, Cutter J, Heng BH, et al. Epidemiology and Control of SARS in Singapore. Ann Acad Med Singap 2006;35:301-16.

Hsien Min Low, ${ }^{1} M B B S, F R C R$, Eugene Low, ${ }^{1} M B B S, F R C R$, Chau Hung Lee, ${ }^{1} M B B S, F R C R$

${ }^{1}$ Department of Diagnostic Imaging, Tan Tock Seng Hospital, Singapore

Address for Correspondence: Dr Eugene Low, Department of Diagnostic Radiology, Tan Tock Seng Hospital, 11 Jalan Tan Tock Seng, Singapore 308133. Email: low_eugene@hotmail.com 